Bull. Mater. Sci., Vol. 17, No. 6, November 1994, pp. 935-950. (C) Printed in India.

\title{
Equation of state of refractory materials at very high temperatures-theoretical and experimental studies
}

\author{
M JOSEPH, N SIVAKUMAR and C K MATHEWS \\ Chemical Group, Indira Gandhi Centre for Atomic Research, Kalpakkam 603 102, India
}

\begin{abstract}
This paper describes some of the theoretical and experimental studies being carried out at IGCAR on the equation of state of refractory materials like reactor fuels at high temperatures $(>3000 \mathrm{~K}$ ). The equation of state is primarily calculated by the principle of corresponding states. The influènce of these equations of state on energy release in a hypothetical core disruptive accident in a fast breeder reactor is indicated. Details of an experimental facility based on laser induced vapourization mass spectrometry, which is being developed to measure the vapour pressures of materials at high temperatures is presented. Possible applications of this facility in other fields of materials research are indicated.
\end{abstract}

Keywords. Equation of state; laser induced vapourization; mass spectrometry; refractory materials; nuclear fuels.

\section{Introduction}

Modern technology demands high temperature and high performance materials such as advanced ceramics, composites and other refractory materials for a variety of applications in space technology (vehicle reentry, missiles etc.), fusion research (first wall material in fusion reactors) and nuclear reactors. These materials often get exposed to very high temperatures $(>2500 \mathrm{~K}$ ) in advanced material processing technologies such as chemical and physical vapour deposition, plasma and laser processing and surface modifications. In order to understand the behaviour of these refractory materials at very high temperatures, it is necessary to establish their equations of state at such elevated temperatures. These properties are especially required to analyse accident conditions.

As an example, let us consider nuclear reactor fuels. Off-normal events in liquid metal cooled fast breeder reactors (LMFBRs) can result in the release of a large amount of energy. Such events have the potential $0 i^{\circ}$ taking the reactor fuel or a portion of it to very high temperatures (up to $5000 \mathrm{~K}$ ) and can lead to partial or complete disassembly of the core. This type of an event is only postulated and hence it is called hypothetical core disruptive accident (HCDA). The analysis of this type of accident requires a knowledge of the equation of state (EOS) of the reactor fuel at high temperatures (IAEA-IWGFR/26 1978). The energy release in such accidents depends strongly on how quickly the vapour pressure develops. The designer has, therefore, to tackle the problem of making accurate estimates of the equation of state of the fuel material. The equation of state for the widely used fuel, namely, $\mathrm{UO}_{2}$ is fairly well known, though based on limited experimental data (Bober et al 1987; Breitung and Reil 1989) and general theoretical models (Fischer 1989). The experimental data on other types of fuels such as carbides, nitrides and metal alloy fuels are not available and theoretical calculations have led only to estimates. 
Theoretical approaches to EOS of such refractory materials are based on the principle of corresponding states (PCS) (Browing et al 1978) and the significant structure theory (SST) (Eyring and John 1969). Both these models have been applied mostly to organic and simple inorgasic systems at temperatures much lower than the region of our interest. They yield parametrized equations whose parameters are obtained by fitting the equations to experimental data. In the case of refractory materials, experimental data are not available at high temperatures. Hence experimental and theoretical studies have to go hand in hand.

Conventional experimental techniques which are used for the measurement of thermophysical properties of refractory materials below $2500 \mathrm{~K}$ cannot be used at ultra high temperatures $(>3000 \mathrm{~K}$ ) because of obvious limitations such as: (i) non-availability of inert container material at such elevated temperatures, (ii) difficulty in attaining and controlling such high temperatures and (iii) difficulty in the measurement of physical and chemical properties under these conditions. For example, the Knudsen effusion technique is often used to measure vapour pressures of refractory materials at low temperatures $(<2500 \mathrm{~K})$. However, this demands that the pressures be in the Knudsen effusion collision-free regime $\left(10^{-4} \mathrm{~atm}\right)$. Under these conditions it is possible to determine the composition of the vapour phase of the system without difficulty. But at high temperatures, the pressures will be much higher, of the order of an atmosphere or higher. Apart from the difficulty of sustaining solid-vapour equilibrium under such high temperature, high pressure conditions, pressure measurement corresponding to the equilibrium temperature and gas sampling are difficult problems. One way of avoiding these problems is by using a dynamic pulse heating technique so that (a) the sample itself acts as a container (therefore no container problem), (b) temperature is generated for a very short duration (order of millisecond or less), limiting energy requirements, and (c) heating is done on a very small sample area and local thermodynamic equilibrium is established at that point during the short duration of heating.

Four different methods of pulse heating, namely, exploding wire technique, electron and neutron pulse heating and laser heating have been employed for this purpose (Ohse et al 1980). Of these, dynamic laser pulse surface heating technique is adopted and developed here-because this method provides a better access to the analysis of the gaseous molecules that are formed during the laser heating of the sample-by using mass spectrometric and spectroscopic techniques (Ohse 1985; Hastie et al 1988; Olender 1990). This will lead to a better understanding of the equilibrium conditions. Hence we have chosen a high power pulsed laser as the heat source to attain these high temperatures in a container-less fashion and a mass spectrometer to characterize the vapour species in order to generate the EOS of technologically important high temperature refractory materials. For this purpose, we have built a laser-induced vapourization mass spectrometer (LIV-MS) in our laboratory.

In this paper we first present the calculation of the equation of state for different fuels, including that of mixed carbide fuel, $\left[\left(\mathrm{U}_{0.3} \mathrm{Pu}_{0.7}\right) \mathrm{C}\right]$, used in the fast breeder test reactor (FBTR), at Kalpakkam, by using the principle of corresponding states. This data is then applied to the study of energy release in a typical hypothetical core disruptive accident (HCDA) in a modularized LMFBR. These results provide some insight into fuel behaviour under such extreme conditions. We will then discuss the LIV-MS system developed in-house for generating the vapour pressure data of reactor fuels at these high temperatures. 


\section{Calculation of the equation of state}

\subsection{Method based on the principle of corresponding states (PCS)}

Because of experimental difficulties, the vapour pressure data for reactor fuels are generally reported only in the low temperature range $(<2500 \mathrm{~K})$. In order to extrapolate these data to very high temperatures we require a theoretical model. The principle of corresponding states (PCS) has been used for this purpose. PCS is based on the assumption that the equation of states of all materials can be generalized on the basis of their reduced temperature $\left(T_{\mathrm{r}}\right)$, reduced pressure $\left(P_{\mathrm{r}}\right)$ and reduced volume $\left(V_{\mathrm{p}}\right)$, the reduced properties of the system being obtained by scaling the actual value in terms of critical value. Thus if the pressure, volume and temperature of one mole of the material are denoted by $P, V$ and $T$ respectively, (their values at critical points being $P_{\mathrm{c}}, V_{\mathrm{c}}$ and $T_{\mathrm{c}}$ ), then the reduced quantities are defined as $P_{\mathrm{r}}=P / P_{\mathrm{c}}, V_{\mathrm{r}}=V / V_{\mathrm{c}}$ and $T_{\mathrm{r}}=T / T_{\mathrm{c}}$. PCS asserts that for all the materials the compressibility factor,

$$
Z=P V / R T \text {, }
$$

is the same function of $V_{\mathrm{r}}, T_{\mathrm{r}}$ and $\alpha$, where $\alpha$ is the Riedel faciur, defined as the slope of the reduced vapour pressure vs temperature curve at the critical point (Browing et al 1978). This method presupposes a knowledge of the critical parameters. An algorithm for finding the critical constants are based on four fundamental generalized equations deduced from experimental data of many $(>70)$ organic compounds as given below (Browing et al 1978):

$$
\begin{aligned}
& 1 / Z_{\mathrm{c}}=3.72+0.26(\alpha-n), \\
& \ln P_{\mathrm{rv}}=\ln T_{\mathrm{r}}-0.0833(\alpha-3.75)\left(36 / T_{\mathrm{r}}-35-T_{\mathrm{r}}^{6}-42 \ln T_{\mathrm{r}}\right), \\
& \rho_{\mathrm{r}}^{1}=1+0.85\left(1-T_{\mathrm{r}}\right)+(1.93+0.2(\alpha-n))\left(1-T_{\mathrm{r}}\right)^{1 / 3}, \\
& 1 / 2\left(\rho_{1}+\rho_{\mathrm{v}}\right)=\mathrm{CT}+\mathrm{D}(\text { law of rectilinear diameter }),
\end{aligned}
$$

where $Z_{c}$ is the $P_{c} V_{c} / R T_{c}$, critical compressibility factor, $\rho_{r}=\rho / \rho_{c}$ the reduced density, $P_{\mathrm{rv}}$ the reduced saturated vapour pressure and $n$, a free parameter obtained from experimental data and for organic fluids it was found to have a value of 7 . Subscripts $c, v, 1$ and $r$ refer to critical point, vapour, liquid and reduced state respectively. $C$ and $D$ are constants of the law of rectilinear diameter used for the calculation of $V_{\mathrm{c}}$ (using estimated $T_{\mathrm{c}}$ and density at melting point).

The estimation of critical constants, $P_{c}, V_{c}, T_{c}$ and $\alpha$, uses (1)-(4) and requires an iterative algorithm for solving these equations for all the critical parameters and $\alpha$. The vapour pressure can be obtained from (2). The details can be found in Browing et al (1978). The input data required are the melting point $\left(T_{\mathrm{m}}\right)$, density at the melting point $\left(\rho_{m}\right)$, volume expansion coefficient $\left(\alpha_{m}\right)$ and vapour pressure at the melting point $\left(P_{\mathrm{m}}\right)$.

The validity of these equations when applied to inorganic liquids has not been established experimentally. In order to ascertain the validity of the PCS method to reactor fuels, we tested it on $\mathrm{UO}_{2}$ which is a relatively well studied system 
(experimental data are available for the input parameters and high temperature vapour pressures reported up to $4500 \mathrm{~K}$ (Bober et al 1987)). The vapour pressure obtained by using the PCS method (with the free parameter $n$, set equal to 7 as reported in the literature (Browing et al 1978) is indicated in figure 1 as PCS1. We can see that the data obtained do not agree well with the experimental values. Hence the value of $n$ was adjusted to match with the available experimental data on the $\mathrm{UO}_{2}$ system and that of liquid alkali metals. When the value of $n$ is changed from 7 (PCS1) to 0.7 (PCS2), the results obtained are in reasonably good agreement with the experimental data (Bober et al 1987), as well as recent in-pile measurements (Breitung and Reil 1989) and calculations based on significant structure theory (Fischer 1989) as can be seen in figure 1.

Even though $\mathrm{UO}_{2}$ is being used widely as a nuclear fuel, a mixed oxide of uranium and plutonium is the fuel of choice in most fast breeder reactors. There is considerable interest in newer types of fuels such as carbides, nitrides and metal alloys of uranium and plutonium on account of their superior properties such as higher density, better thermal conductivity and favourable neutronic properties. In

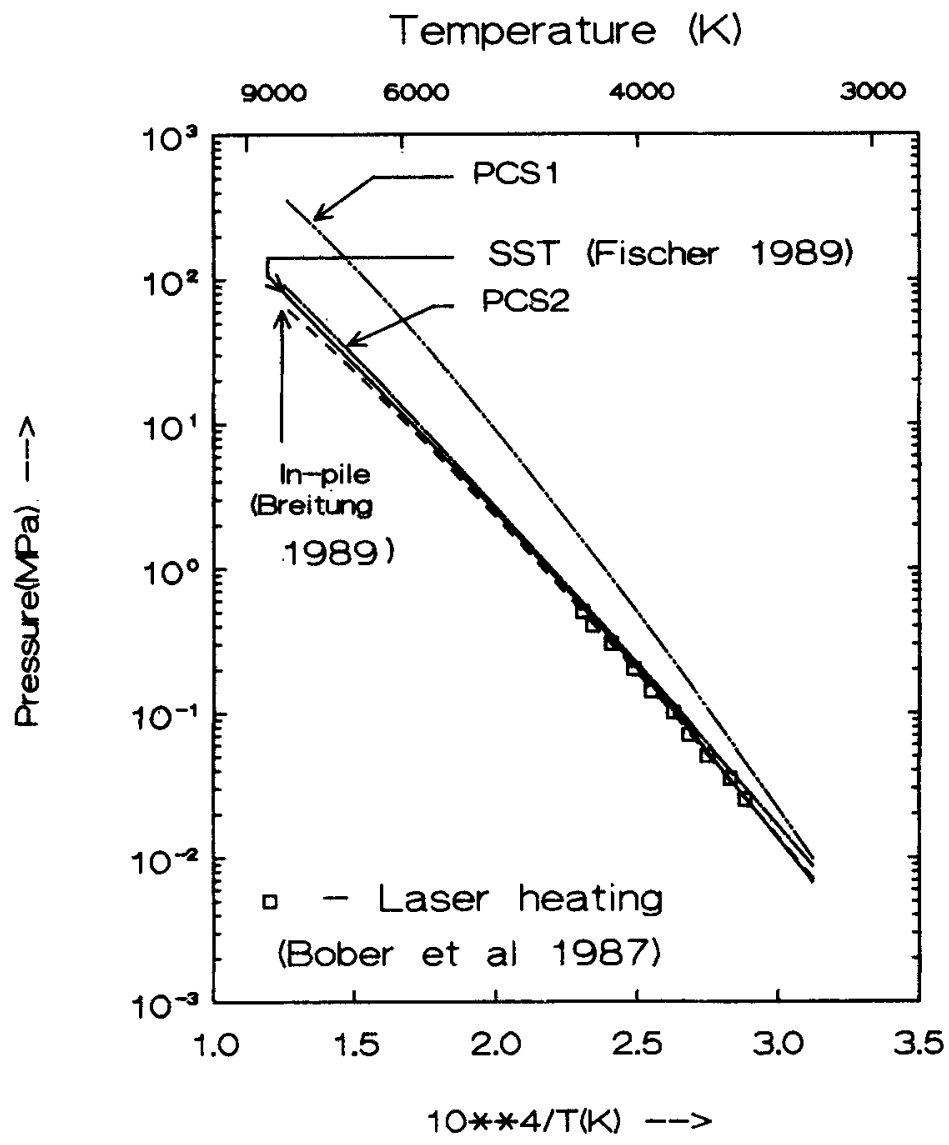

Figure 1. Comparison of vapour pressure of $\mathrm{UO}_{2}$ in the liquid region by different methods. 
fact, the FBTR at Kalpakkam employs a mixed carbide fuel. Hence, the above model (PCS2) was applied to all possible fuel types, namely $\mathrm{UC}, \mathrm{UO}_{2}$, mixed carbide $\left[\left(\mathrm{U}_{0.3} \mathrm{P}_{0.7}\right) \mathrm{C}\right]$, metal alloy $\left[\left(\mathrm{U}_{0.6} \mathrm{Pu}_{0.17} \mathrm{Zr}_{0.23}\right)\right]$, UN, mixed nitride $\left[\left(\mathrm{U}_{0.8} \mathrm{Pu}_{0.2}\right) \mathrm{N}\right]$ and PuN. Wherever experimental data were not available at the required melting point, reasonable estimates were made by employing strategies like extrapolation of low temperature data in order to arrive at the input parameters. The input parameters used in the computation are shown in table 1. The accuracy of the computed equations of state is limited by the input data. Nevertheless, the results bring out trends which are useful in making some broad conclusions. The vapour pressures of different fuels of interest in reactor calculations, in the temperature range from $3000 \mathrm{~K}$ to $6000 \mathrm{~K}$, are plotted in figure 2 . It is seen that UC has the lowest vapour pressure while PuN has the highest (for $T>4000 \mathrm{~K}$ ). It must be mentioned here that UN and PuN are not reactor fuels, but only fuel materials. The mixed nitride which is a potential fuel, has lower vapour pressure than UN and PuN. Some vapour pressure curves cross each other indicating that even if one system has relatively higher pressure at low temperatures, the trend can reverse at high temperatures. The values of critical constants obtained are shown in table 2. A sensitivity study on the input data showed that the volume expansion coefficient is the most sensitive input parameter (Joseph et al 1989).

\subsection{Extrapolation method}

No experimental data is available on the mixed carbide fuel of the composition that is being used in FBTR (Ganguly et al 1988). The low temperature (below melting point) vapour pressure data, for the vapour species $\mathrm{Pu}(\mathrm{g}), \mathrm{U}(\mathrm{g}), \mathrm{UC}_{2}(\mathrm{~g})$, $\mathrm{C}_{1}(\mathrm{~g}), \mathrm{C}_{2}(\mathrm{~g}), \mathrm{C}_{3}(\mathrm{~g})$ and $\mathrm{C}_{4}(\mathrm{~g})$ have been computed in our laboratory by thermodynamic modeling (Joseph et al 1989). The pressure-temperature data thus obtained is valid only up to temperatures less than the melting point of the carbide and hence this has to be extrapolated across the melting point to temperatures upto $5000 \mathrm{~K}$. A simple and reasonable approach to this extrapolation is to correct the slope of the partial pressure curve of each species for the heat of fusion. Such extrapolation was found to give good agreement with the literature data as well as with the modified PCS, as discussed above, for $\mathrm{UO}_{2}$ (up to $5000 \mathrm{~K}$ ). Similarly, good agreement is seen in the case of UC as well (see figure 3). But for the FBTR fuel the agreement between the above extrapolation and the modified PCS method is rather poor (see figure 4), differing by an order of magnitude. This is not

Table 1. Input parameters for PCS.

\begin{tabular}{lccccl}
\hline Fuel & $T_{\mathrm{m}}(\mathrm{K})$ & $P_{\mathrm{m}}(\mathrm{atm})$ & $\rho_{\mathrm{m}}(\mathrm{g} / \mathrm{cc})$ & $\alpha_{\mathrm{m}} * e^{-6} / \mathrm{K}$ & \\
\hline $\mathrm{UO}_{2}$ & 3115 & $4 \mathrm{E}-2$ & 8.74 & 103 & $\mathrm{~m}$ - melting point \\
UC & 2780 & $2.8 \mathrm{E}-5$ & 9.84 & $78 \cdot 1$ & $\alpha$ - vol. exp. coft. \\
UN & 3035 & $6.0 \mathrm{E}-1$ & 11.41 & 68.2 & $\rho-$ density \\
Alloy & 1550 & $1.3 \mathrm{E}-8$ & 13.75 & 74.6 & \\
FBTR & 2400 & $1.1 \mathrm{E}-3$ & 10.0 & 77.4 & \\
PuN & 2993 & $1.7 \mathrm{E}-1$ & 10.76 & 117 & \\
MN & 3053 & $8.8 \mathrm{E}-2$ & 11.36 & 68.4 & \\
\hline
\end{tabular}




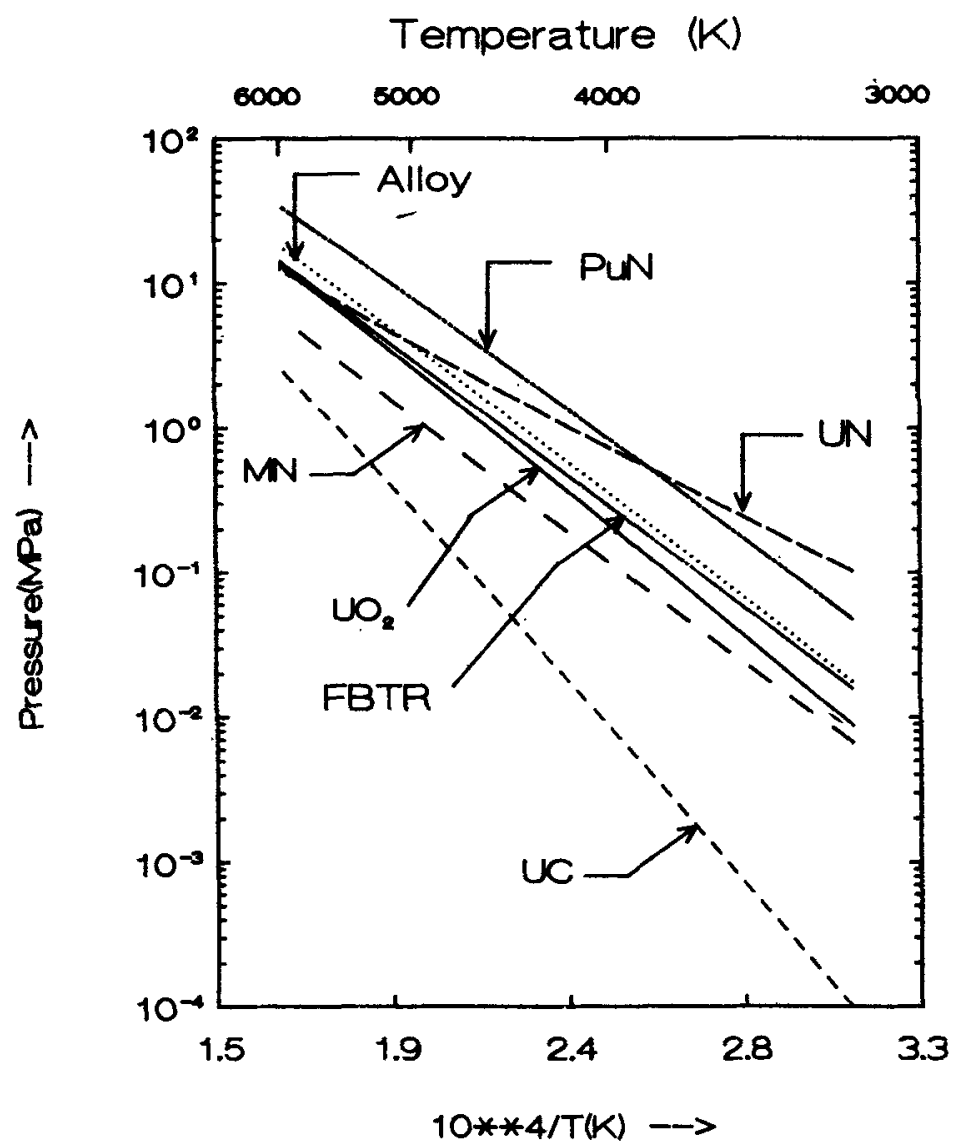

Figure 2. Vapour pressure of different types of reactor fuels in the temperature region of 3000 to $6000 \mathrm{~K}$.

Table 2. Critical parameters for different fuels by PCS.

\begin{tabular}{lrrrrrr}
\hline & & & & \multicolumn{3}{c}{$\log P(\mathrm{MPa})=A-B e 4 / T+C \log (T)$} \\
\cline { 6 - 7 } Fuel & $T_{\mathrm{c}}(\mathrm{K})$ & $P_{\mathrm{c}}(\mathrm{atm})$ & $\rho_{\mathrm{c}}(\mathrm{g} / \mathrm{cc})$ & $\mathrm{A}$ & $\mathrm{B}$ & $\mathrm{C}$ \\
\hline UO2 & 8098 & 1017 & 2.12 & 11.23 & 2.478 & -1.58 \\
UC & 9759 & 1349 & 2.24 & 17.00 & 3.564 & -2.82 \\
UN & 10383 & 2034 & 2.85 & -4.23 & 1.077 & 1.89 \\
Alloy & 8928 & 2227 & 3.09 & 8.18 & 2.222 & -0.85 \\
FBTR & 9221 & 1403 & 2.31 & 5.13 & 2.050 & -0.15 \\
PuN & 7329 & 1227 & 2.66 & 10.91 & 2.24 & -1.50 \\
MN & 10594 & 1942 & 2.75 & 2.18 & 1.93 & 0.48 \\
\hline
\end{tabular}

surprising as the input data is not known very reliably. Extrapolation is from computed partial pressures. As for PCS, input data such as density, volume expansion coefficient and vapour pressure, all at melting point are only estimates. This points to the need for experimental data. 
Equation of state of refractory materials at high temperatures

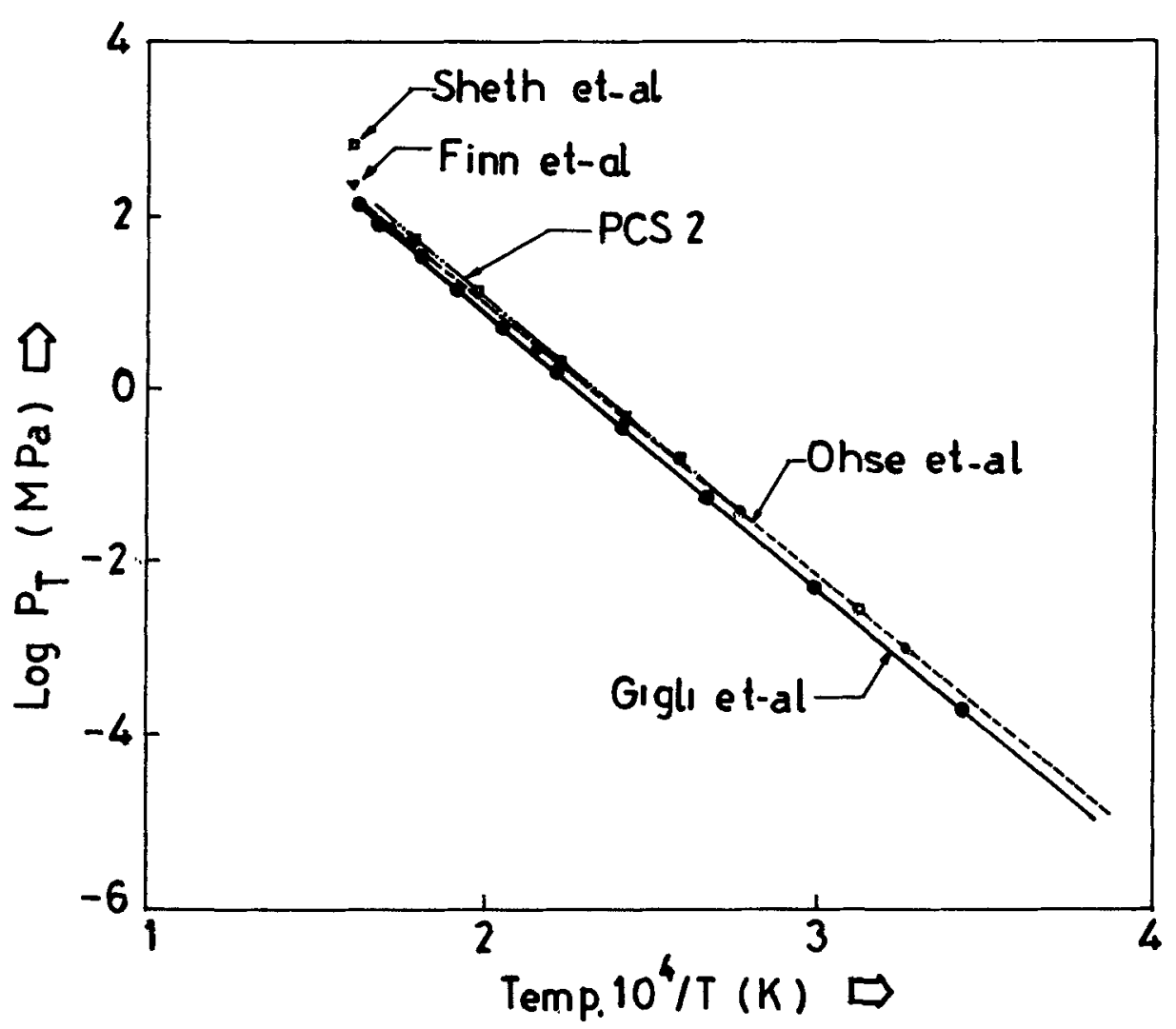

Figure 3. Vapour pressure over uranium monocarbide $(3000-9000 \mathrm{~K})$.

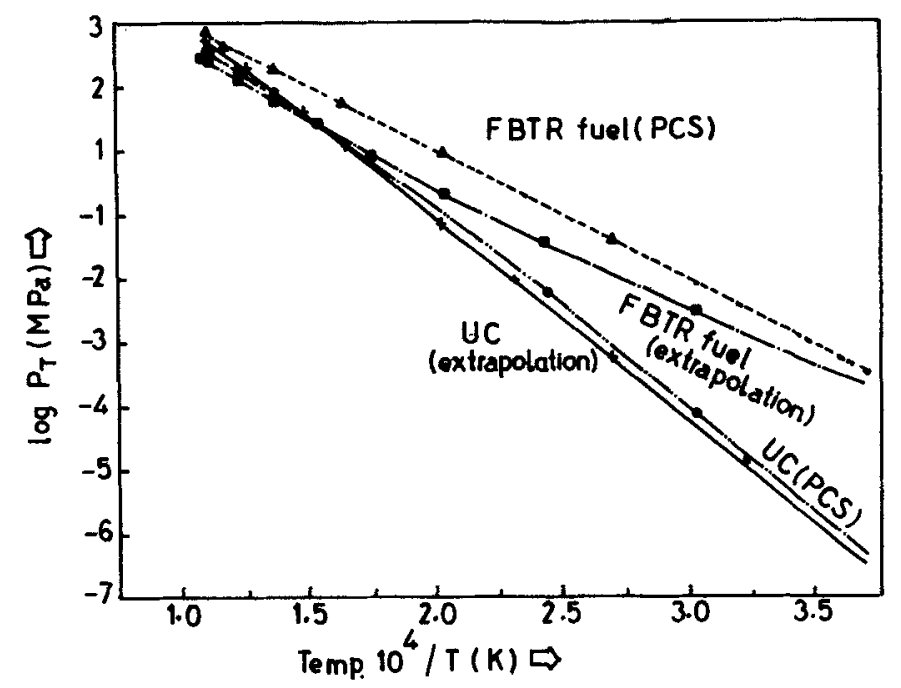

Figure 4. Vapour pressure over uranium monocarbide and mixed carbide $(3000-9000 \mathrm{~K})$. 


\section{Influence of fuel type on the HCDA energetics}

The EOS has a strong impact on the magnitude of energy release in an HCDA. In this accident scenario, the power excursion and the energy release are terminated only by core disassembly due to pressure generated on account of the heating of the fuel. Assuming typical initial reactor conditions and those corresponding to a typical unprotected loss of flow accident in a medium sized LMFBR (PFBR-500 being designed in India) for all the fuel types, the core disassembly analysis was performed (Joseph M, Bhaskar Rao P and Mathews C K, to be published) by using the VENUS code (Jackson and Nicholson 1972). The results obtained for the energy release, work potential and peak fuel temperature reached are shown in figure 5. It can be seen that the fuel can reach a peak temperature of around $4500 \mathrm{~K}$ and that in that region PuN has the highest vapour pressure and UC the lowest. Correspondingly, the energy release is the highest $(6554 \mathrm{MJ}$ thermal and $232 \mathrm{MJ}$ mechanical) in the case of UC and the lowest for PuN (4353 MJ, thermal and 18 MJ, mechanical).

\section{Experimental facility for EOS}

As indicated above, theoretical tools such as PCS or SST are tested for their validity only for low temperature systems such as organic liquids and simple
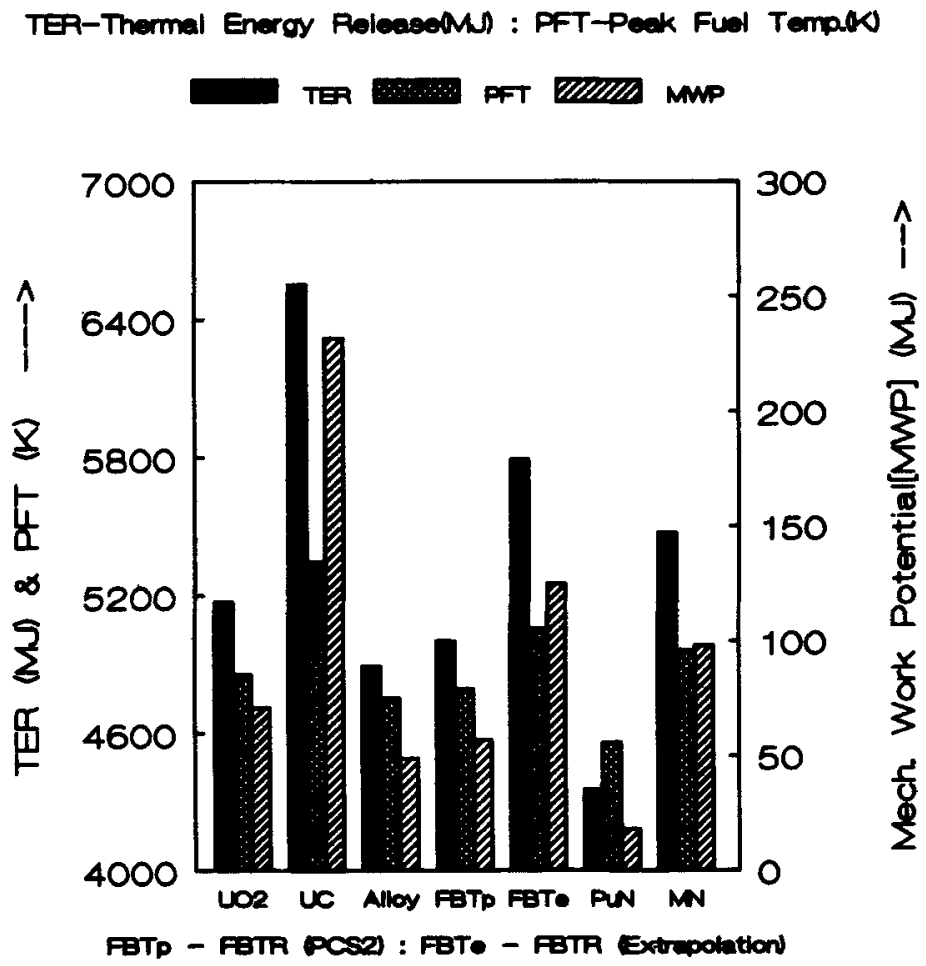

Figure 5. Results of HCDA for different types of fuels. 
inorganic materials. They are not tested for refractory materials mainly because of experimental difficulties. We have set up an LIV-MS system to measure the vapour pressure of some of these materials at high temperatures.

The LIV-MS facility primarily consists of a high power pulsed laser which acts as a heat source and a mass spectrometer to characterize the vapour species. A schematic of the experimental facility is shown in figure 6. It is based on three vacuum chambers, of which only two are shown in the figure. The first chamber is pumped by a $2000 \mathrm{l} / \mathrm{sec}$ capacity $10 \mathrm{inch}$, Edwards diffusion pump (model Mk $2 / 2000$ ). The second chamber is pumped by a $1500 \mathrm{l} / \mathrm{sec}$ capacity cryo pump (Leybold AG, Model RW2 compressor) and the third by a turbo pump with a capacity of $1000 \mathrm{l} / \mathrm{sec}$ (Leybold, Turbovac $1000 \mathrm{~W} / \mathrm{L}$ ). The three chambers are aligned to have the same axis (that of the molecular beam) by employing a $\mathrm{CW}$ $\mathrm{He}-\mathrm{Ne}$ laser. A skimmer separates the first and second chambers whereas a collimator separates the second and third chambers. Because of the good system conductance, the background pressure is very low $\left(<10^{-6}\right.$ torr). Small conductances between the chambers allows for differential pumping. In the first chamber, the vaccum condition permits the establishment of a high gas expansion pressure ratio and there is minimal perturbation of the vapour plume by post-expansion gas collisions with the background gas molecules. (The laser vapourization time is too short, of the order of few $\mu \mathrm{sec}$ for the ionization gauges to respond).

\subsection{The mass spectrometer}

The mass analyser used is a quadrupole mass filter (Extrel Corp.) with a mass range of 1 to $700 \mathrm{amu}$. This is housed in the second chamber such that its axis is perpendicular to the beam path (i.e. crossed beam configuration). The molecular beam coming through the skimmer gets ionized in the ion source of the mass spectrometer. These ions are then accelerated into the quadrupole. The DC and RF voltages of the quadrupole mass filter (QMF) are so adjusted as to allow only

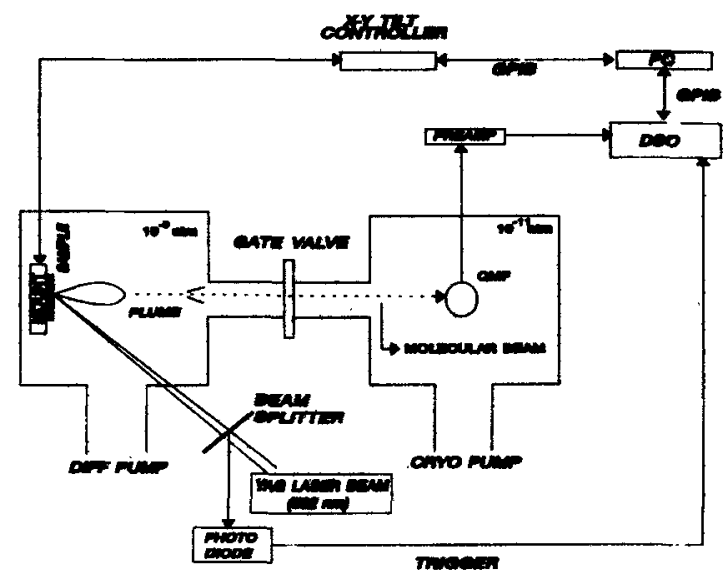

Figure 6. Schematic of LIV-MS facility. 
ions of a given me to pass through. Mass scan is achieved by varying the DC voltage, while keeping the DC/AC voltage ratio to be constant. The ions are detected by an electron multiplier and the signal is amplified by an analog charge preamplifier. The whole QMF assembly is mounted on an 8 inch UHV flange fixed to one of the side ports of the second chamber.

\subsection{The sample positioning system}

The sample is placed in the first chamber by mounting it on a kinematic stage (see figure 6). This stage movement is controlled by four DC drive motors, two for axial translation motion and two for tilts in different directions. The range of the translation motion is $25 \mathrm{~mm}$ in either direction and tilt is $\pm 7^{\circ}$ (M/s Physik Instruments, Model C-804). The movement of these motors are controlled remotely through a personal computer (PC). This system allows the sample to be positioned or rastered, as desired, between laser shots. For uniform plume generation, the sample has to be rastered, otherwise a crater of the order of $200-300 \mu \mathrm{m}$ in diameter will be formed at the laser focusing point and this in turn will decrease the plume intensity. Hence without rastering the sample, we cannot get good reproducibility in ion intensity. A typical raster rate of $100 \mu \mathrm{m} / \mathrm{sec}$ provides more than $90 \%$ shot-to-shot overlap at $10 \mathrm{~Hz}$ laser pulsing with a focused spot diameter of $\sim 300 \mu \mathrm{m}$. Figure 7 shows the crater formation on $\mathrm{UO}_{2}$ pellet for about 1000 laser shots on the same focusing point.

\subsection{The laser system}

Different types of lasers have been used for the evaporation of refractory materials (Ready 1971; Hastie et al 1988; Olender 1990). We have chosen a Nd-YAG laser

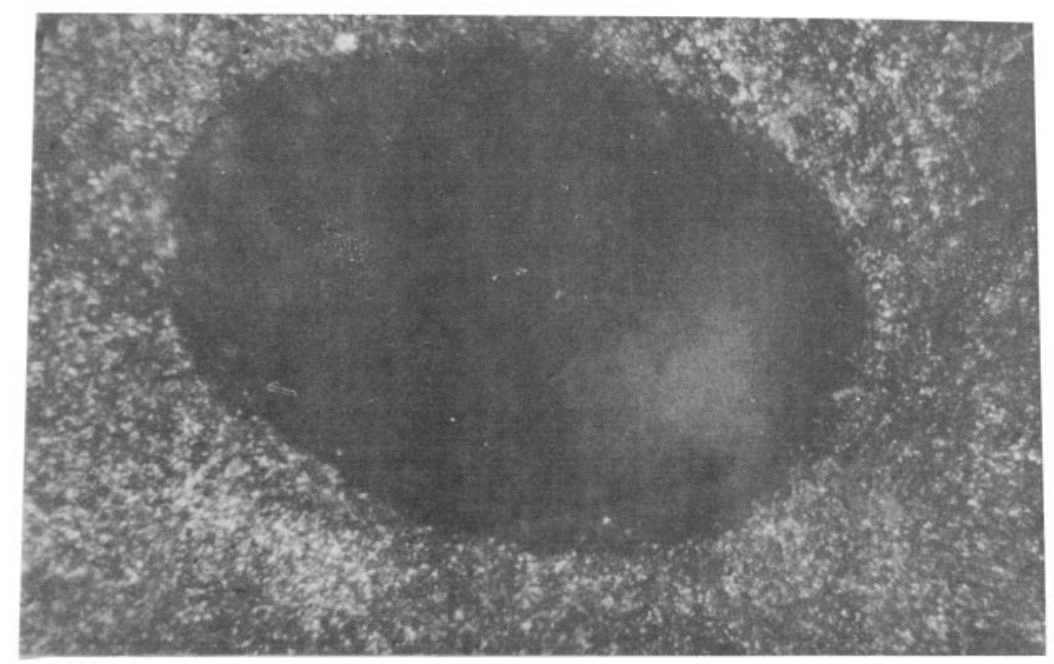

Figure 7. Micrograph of crater formed on $\mathrm{UO}_{2}$ pellet for about 1000 laser shots. Crater size: about $350 \mu \mathrm{m}$ dia and $25 \mu \mathrm{m}$ deep. 
system (Continuum, Model NY-61-10) to suit our experimental conditions. The maximum beam energy is $600 \mathrm{~mJ}$ at $1064 \mathrm{~nm}$ with $5-7 \mathrm{nsec}$ pulse width. The laser is operated in the Q-switched mode at a repetition rate of $10 \mathrm{~Hz}$. The laser beam spatial profile is a Gaussian distribution $\left(\mathrm{TEM}_{(\mathrm{k})}\right)$. The laser system is equipped with mixing crystals to provide additional wavelengths at $532 \mathrm{~nm}(300 \mathrm{~mJ}), 355$ $\mathrm{nm}(170 \mathrm{~mJ})$ and $266 \mathrm{~nm}(50 \mathrm{~mJ})$. A laser fluence of $20-40 \mathrm{~mJ} / \mathrm{cm}^{2}$ at $532 \mathrm{~nm}$ was found to be sufficient to generate a reasonably good vapour plume on ceramic materials (Hastie et al 1990). In our experiments we have used $\sim 50 \mathrm{~mJ}$ pulses. The laser beam is directed to the target sample, through a quartz view port, by using suitable dielectric coated mirrors for beam steering and a $250 \mathrm{~mm}$ focal length quartz lens for focusing. The power density generated is of the order of $10^{9} \mathrm{~W} / \mathrm{cm}^{2}$.

\subsection{Data acquisition}

The ions of interest falling on the detector gives out an analog signal. This signal from the detector is fed to a digital storage oscilloscope (DSO) as indicated in figure 6. In this work we have used a LeCroy model $9450 \mathrm{~A} 300 \mathrm{MHz}$ two channel oscilloscope. This has a $50 \mathrm{~K}$ storage memory for each channel with a sampling rate of $400 \mathrm{Ms} / \mathrm{S}$. The laser beam is partially split, before it enters into the vacuum chamber. The less intense of the split beam is made to fall on a photodiode (UT Sensors Inc, Model PIN-HR040). The signal from the photodiode is used to trigger DSO to start the data collection process.

\subsection{Measurement}

In the actual experiment, the sample position is adjusted to the location of interest and the laser beam is focused. After this the sample is rastered. The plume formed in each laser pulse is skimmed into the second chamber and the molecules in the beam are ionized in the QMF ion source. The mass spectrometer is tuned to the mass of interest (single ion mode), so that the signal is seen on the scope (DSO). In order to get a good signal-to-noise ratio, averaging of the signal is done over 100 to 1000 laser shots. Generally, the collection of the signal for about $5 \mathrm{msec}$ from the start of the laser pulse is found to be sufficient to get the time of arrival (TOA) profile of each ion mass. This $5 \mathrm{msec}$ period includes the time for evaporation of the order of few $\mu \mathrm{sec}$, transit of the molecular beam for about $50 \mathrm{~cm}$ (the distance from the evaporation surface to the ion source), and electronic detection, including RC time constant. Figure 8 shows a typical mass selected profile of ion intensity for $\mathrm{UO}_{2}(\mathrm{~g})$ species. The data collected in the DSO is transferred to a computer for further processing. By tuning the QMF for different masses of interest, the intensity of each species which is formed in the laser evaporation can be obtained.

\section{Studies on $\mathrm{UO}_{2}$ pellet}

A $\mathrm{UO}_{2}$ pellet obtained from the Nuclear Fuel Complex, Hyderabad, was subjected to laser irradiation. The species $\mathrm{O}, \mathrm{U}, \mathrm{UO}$ and $\mathrm{UO}_{2}$ were identified in the vapour 


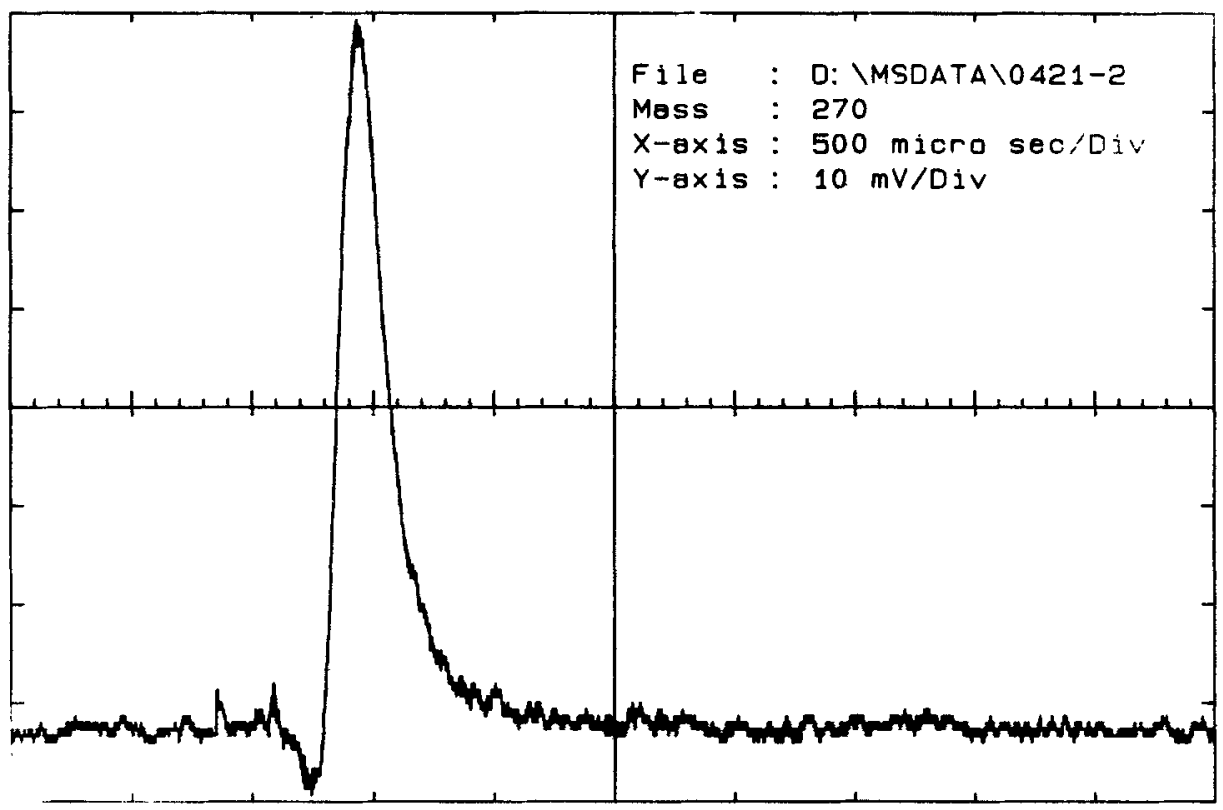

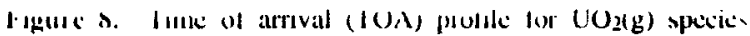

phase by means of the QMF. If these species are in thermal equilibrium with each other, say at temperature $T$, then according to the kinetic theory of gases,

i.e.

$$
1 / 2 M v^{2}=3 / 2 K T
$$

$$
\begin{aligned}
& 1 / 2 M(d / /)^{2}=3 / 2 K T, \\
& t \propto \sqrt{M / T}
\end{aligned}
$$

wl se, $M$ is molecular weight of the individual species, $K$ the Boltzman constant and $t$ is the lime of arrival ( 10.1$)$ as shown in figure 8 . Hence, a plot of the time of arrival as a function of the square root of molecular weight should yield a straight line. Figure 9 shows the plot obtained in the present experiment. The straight line nature of the plot shows that the gas species are in thermal equilibrium with each other, at least with respect to translational energy. The temperature which can be obtained from the slope of the lin ar $p_{\text {. }}$ 't corresponds to a cool beam, which results from the supersonic expansion of the plume.

In laser evaporation, the plume formation s ' $n$ be considered to be similar to the beam formation process of supersonic expansi. ns through a nozzle, where the beam composition after the expansion will be same as that of the pre-expansion temperature and partial pressure conditions (Bonrell and Hastie 1979). The hyh pumping speed of the vacuum system and the pulsed nature of vapour evolutw11 process also ensure the production of high expansion ratios, which is a necessary :ondition for 'freving in' the species distribution. 


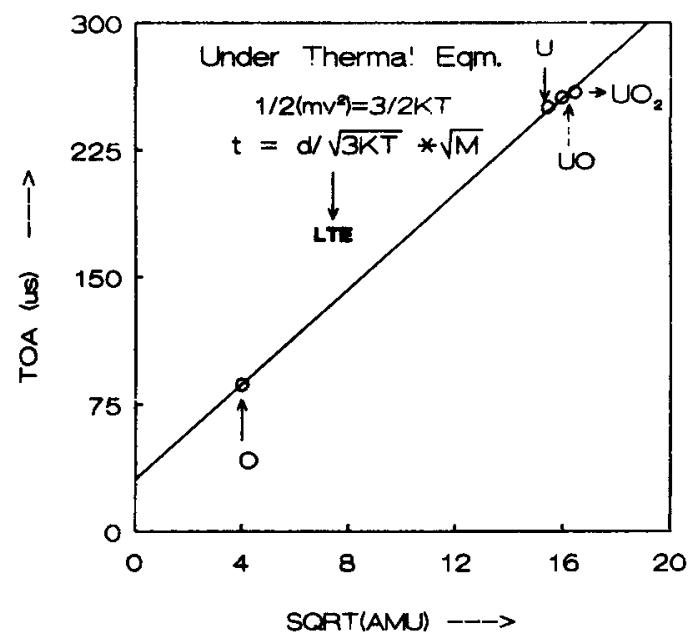

Figure 9. Dependence of TOA for $\mathrm{O}, \mathrm{U}, \mathrm{UO}, \mathrm{UO} 2$ vapour species as a function of their molecular weight (square root).

Once the existence of thermal equilibrium is established, then there should be an equilibrium surface temperature. The molecular composition in the expanded beam is the same as that of the composition at the surface. Hence, if the surface temperature is known, then the partial pressure of the vapour species can be determined by the relation,

$$
p_{\mathrm{i}}=K I_{\mathrm{i}} T
$$

W here $p_{\mathrm{i}}$ and $t_{\mathrm{i}}$ are the partial pressure and intensity of the species $\mathrm{i}$, and $K$ the calibration constant. Physical measurement of surface temperature for the interaction of laser pulses of nanosec duration is beyond the state of art. Hence indirect methods are employed, such as the comparison of TOA of the species of the system with that of a relatively well studied reference such as graphite (Hastie et al 1988b). For example, if $t_{\mathrm{c}}$ is the TOA of $\mathrm{C}_{2}(\mathrm{~g})$ over the graphite (reference) sample having a surface temperature $T_{\mathrm{g}}$ and $t_{\mathrm{u}}$ is the TOA of $\mathrm{UO}_{2}(\mathrm{~g})$ over the $\mathrm{UO}_{2}$ sample with a surface temperature, $T_{u}$, then under identical experimental conditions,

$$
T_{\mathrm{u}}=T_{\mathrm{g}}^{*}\left(t_{\mathrm{u}} / \sqrt{M_{\mathrm{u}}}\right) /\left(t_{\mathrm{c}} / \sqrt{M_{\mathrm{c}}}\right),
$$

where $M_{u}$ and $M_{\mathrm{c}}$ are the molecular weights of $\mathrm{UO}_{2}(\mathrm{~g})$ and $\mathrm{C}_{2}(\mathrm{~g})$ respectively. Such studies on the determination of temperature and pressure are in progress.

\section{Other applications}

Though the primary motivation for building an LIV-MS system in our laboratory was the experir:ental determination of the equation of state of reactor fuels, sufficient flexibility was built into the experimental assembly so that it can be used in a variety of applications. Some of these are indicated below. 
The results reported are based on a quadrupole mass spectrometer. In addition to this a reflection type time-of-flight mass spectrometer (TOFMS) is under construction for incorporation into the system. TOFMS has several inherent advantages such as pulsed operation, the acquisition of the entire spectrum for every laser pulse, high resolution, large mass range and high transmission.

In a linear TOFMS (Wiley and McLaren 1955), the resolution is limited on account of the energy spread of the ion packet. In the reflection type TOFMS (RTOFMS), which was originally developed by Mamyrin et al (1973), the resolution was improved to several thousands. This is achieved by reflecting the ions back by means of an appropriate grid of electrodes. The ions with the same $\mathrm{m} / \mathrm{e}$ ratio but larger in kinetic energy penetrate deeper into the reflecting field and take longer time to turn around than ions of lower energy. The electrostatic potentials are adjusted so that the fast ions lag behind the slow ions after reflection, but catch up with the slow ions at the point of detection after the second drift region. Thus by compensating for kinetic energy spread, bunching (narrowing down the spread in the arrival time), of ions of each mass-to-charge ratio is achieved at the detector. We have designed such a RTOFMS (see figure 10) and this will be put into use shortly. The incorporation of RTOFMS in the LIV-MS system significantly extends the range of application of this experimental facility. Some of these applications are indicated below.

\subsection{Cluster studies}

Clusters represent an intermediate state of matter between small molecules and the condensed phase. Research in the chemistry and physics of clusters is currently developing at a rapid pace. It was the introduction of laser vapourization technique for the production of supersonic metal cluster beams by Smalley and co-workers (Kroto et al 1985), that led to the discovery of fullerenes. There is a considerable interest in the scientific world in studying gas phase metal clusters in order to

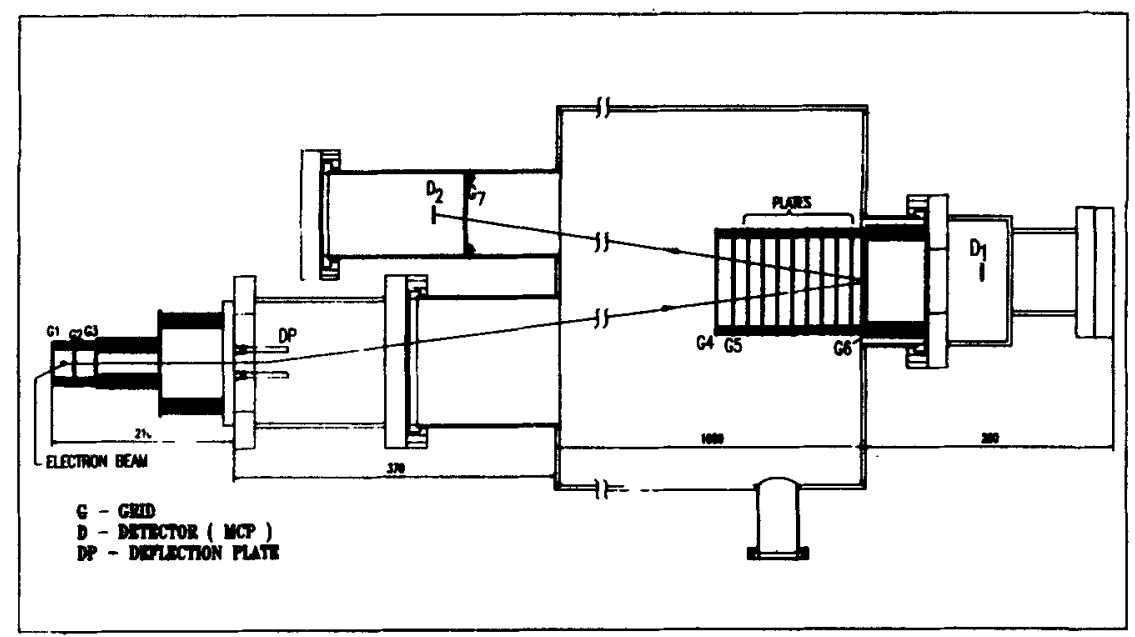

Figure 10. Schematic of reflection time-of-flight mass spectrometer (RTOFMS). 
understand the nature of bonding, electronic structure and a host of other properties. In particular there is a strong interest in the variation of electronic structure, especially the band gap, from small clusters to the condensed phase.

One method of generating clusters of refractory materials is to vapourize the material by means of a pulsed laser and to sweep the resulting plume by means of an inert gas stream adiabatically expanding from high pressures through a molecular beam valve. Combined with a high resolution, high mass range mass spectrometer such a system can be used to study a wide range of clusters. The laser-TOFMS system is being used extensively in these type of studies. For example, when graphite was vapourized by a laser beam, the largest cluster size observed was $\mathrm{C}_{9}$ (Hastie et al 1988b) (with $\mathrm{C}_{3}$ being the major species). But, in the same vapourization experiment, when the plume was swept by a $\mathrm{He}$ gas jet, much larger clusters were observed, the most dominant of them being $C_{60}$ and $C_{70}$ (O'Brien et al 1988). Buckminsterfullerene or $C_{60}$, which has been the subject of extensive research, was identified to be orders of magnitude more abundant than all other clusters of atoms. Metal clusters show remarkable stability at magic numbers. Both metal and semiconductor clusters are under investigation with a view to understanding the transition from cluster to bulk behaviour like metallic or semiconductor properties. Our LIV-MS system is equipped with a pulsed molecular beam valve to enable us to take up cluster studies.

\subsection{Surface analysis}

The measurement of the local chemical composition at the surfaces and interfaces are currently being done using techniques such as Auger electron spectroscopy (AES) and secondary ion mass spectrometry (SIMS). The practical limitation of these techniques, namely, sensitivity (AES) and matrix effect (SIMS) are overcome, to a larger extent, by the recently reported surface analysis by laser ionization (SALI) technique (Welkie et al 1990). In SALI a monolayer of the sample surface is sputtered or desorbed by a probe beam (ions, electrons, laser) and a UV laser beam is directed through the plume of the sputtered neutrals, immediately above the sample surface, so as to obtain saturated ionization. Because ions are produced by a pulsed laser, subsequent mass analysis is done by TOFMS. Hence with minor modification in the experimental configuration, our system can be used for such chemical mapping studies.

\subsection{Thin film coating}

For the preparation of thin films of refractory material, laser induced evaporation and deposition is a relatively simple and clean method. This method has many practical advantages (Sankur and Hall 1985). Preliminary experiments have been done to prepare a good quality tin oxide film, which has wide applications as a semiconductor sensor for the detection of inflammable gases.

When highly selective and sensitive detection of ions is required under almost background-free condition, we can use resonance ionization mass spectrometry (RIMS) (Nogar et al 1985). This LIV-MS facility can also be used for the analysis of macro molecules such as proteins, peptides etc by using the matrix assisted laser desorption and ionization (MALDI) (Karas and Hillenkamp 1989) method. 


\section{Conclusions}

Theoretical and experimental approaches to obtain the equation of state of refractory materials like reactor fuels at very high temperatures are discussed. Theoretical models have given results which can be used for making an estimate of the behaviour of reactor fuels under hypothetical core disruptive accidents. An experimental facility based on laser induced vapourization combined with mass spectrometry has been set up to generate the vapour pressures of these materials in the temperature range $2500-5000 \mathrm{~K}$. Preliminary results are presented. Possible application of this facility in many other fields of research such as materials science and engineering are indicated.

\section{Acknowledgements}

The authors are grateful to Dr P Bhasker Rao for carrying out the energy release calculations. They also acknowledge the help rendered by Shri D Darwin Albert Raj in installing the quadrupole mass spectrometer and the many fruitful discussions with Dr K S Viswanathan during the course of this work.

\section{References}

Bober M, Singer J and Trapp M 1987 Nucl. Sci. Eng. 97344

Bonnell D W and Hastie J W 1979 Transpiration mass spectrometry of high temperature vapours, Proc. 10th metals res. symp. (Washington DC: US Govt. Printing Office) p. 537

Breitung W and Reil K O 1989 Nucl. Sci. Eng. 10126

Browing P, Gillan M J and Potter P E 1978 Rev. Int. Hautes Temp. Refract. Fr. 15333

Eyring H and John M S 1969 Significant liquid structure (New York: John Wiley \& Sons Inc.)

Ganguly C, Jain C G, Ghosh J K and Roy P R 1988 J. Nucl. Mater. 153178

Fischer E A 1989 Nucl. Sci. Eng. 10197

Hastie J W, Bonnell D W and Schenck P K 1988a High Temp. Sci. 25117

Hastie I W, Bonnell D W and Schenck P K 1988b High Temp. High Press. 2073

Hastie J W, Bonnell D W, Schenck P K and Joseph M 1990 Proc. of symp. on high temperature chemistry (Pennington, NJ, USA: Electrochemical Soc. Meeting)

IAEA-IWGFR/26 1978 Summary report of specialists meeting on equation of state of materials of relevance to the analysis of hypothetical fast reactor accidents, IAEA, Vienna

Jackson J F and Nicholson R B 1972 Report ANL-7951, Argonne

Joseph M, Mathews C K and Bhaskar Rao P 1989 J. Nucl. Mater. 168220

Karas M and Hillenkamp F 1989 Adv. Mass Spectrom. 11416

Kroto H W, Heath J R, O'Brien S C, Curl R F and Smalley R E 1985 Nature (London) 318162

Mamyrin B A, Karataev V I, Shmikk D V and Zagulin V A 1973 Sov. Phys. JETP 3745

Nogar N S, Downey S W and Miller C M 1985 Anal. Chem. 571144

O'Brien S C, Heath J R, Curl R F and Smalley R E $1988 \mathrm{~J}$. Chem. Phys. 88220

Ohse R W $1985 \mathrm{~J}$. Nucl. Mater. 130165

Ohse R W, Babelot J F, Frezzotti A, Long K A, Magill J, Cercignani C and Scotti A 1980 High Temp. Sci. 1335

Olender D R 1990 High Temp. Sci. 27411

Ready J F 1971 Effects of high power laser radiation (New York: Academic Press)

Sankur H and Hall R 1985 Appl. Opt. 243343

Welkie D G, Daiser S and Becker C H 1990 Vacuum 411665

Wiley W C and McLaren I H 1955 Rev. Sci. Instrum. 261150 